\title{
Calculation of energy states of excitons in square quantum wells
}

\author{
(C) P.A. Belov \\ Department of Computational Physics, St. Petersburg State University, \\ 198504 St. Petersburg, Russia \\ E-mail: pavelbelov@gmail.com
}

The ground and excited energy states of excitons in single square GaAs-based quantum wells are found by the numerical solution of the three-dimensional Schrödinger equation.This equation is obtained within the envelope-function formalism from the exciton energy operator using the spherical approximation of the Luttinger Hamiltonian. Precise results for the exciton states are achieved by the finite-difference method. The radiative decay rates of the calculated states are also determined.

\section{Acknowledgments}

The work is supported by SPbU (grants No.11.38.213.2014 and No 11.38.241.2015), RFBR (grants No 16-02-00245, No 16-32-00047) and DFG in the frame of Project ICRC TRR 160. The calculations were carried out using the facilities of the SPbU Resource Center „Computational Center of SPbU“. 